\title{
Missing Edward Scissorhands
}

\author{
Author: Nida Butt ${ }^{\mathrm{A}}$
}

\section{Introduction}

A 59-year-old woman presented to the acute stroke service in February 2019 as a thrombolysis call. She had suddenonset right arm and leg weakness and inability to speak, witnessed by her neighbour who saw her collapsing and called the paramedics. She had a past medical history of multiple ischaemic strokes (right frontal infarct in April 2016, right thalamic infarct in March 2016 and bilateral occipital infarcts in 2011). This was against a background of several cardiovascular risk factors such as hypertension, hypercholesterolaemia, peripheral vascular disease, ischaemic heart disease with tissue aortic valve replacement, autoimmune hypothyroidism and a longstanding smoking history. Her regular medications included dual antiplatelet agents, bisoprolol, amlodipine, atorvastatin, ranitidine and levothyroxine. Clinical examination was consistent with a severe left middle cerebral artery stroke with a National Institutes of Health Stroke Scale (NIHSS) score of 28. Computed tomography (CT) of the head and CT angiography excluded intracranial bleed and a proximal M1 thrombus respectively, hence she was thrombolysed with a door-to-needle time of 46 minutes. She recovered remarkably well from her limb weakness but remained profoundly aphasic.

\section{Materials and methods}

An electronic patient records system called Evolve was used to review all previous admissions and clinic attendances for the patient. A picture archiving and communication system and integrated clinical environment were used to look at radiological investigations and blood results respectively.

\section{Results and discussion}

This patient had first presented to the stroke service in 2011 with strokes in different vascular territories. It was noted that she had thrombocytopenia and raised creatinine during that episode, as well as at the time of all subsequent strokes

(Figs 1 and 2). A thorough workup to find the aetiology of stroke was carried out that included normal carotid Doppler, R-test and transoesophageal echocardiography (TOE). She had negative screening results for thrombophilia, vasculitis and

Author: ${ }^{A}$ Oxford University Hospitals NHS Foundation Trust, Oxford, UK

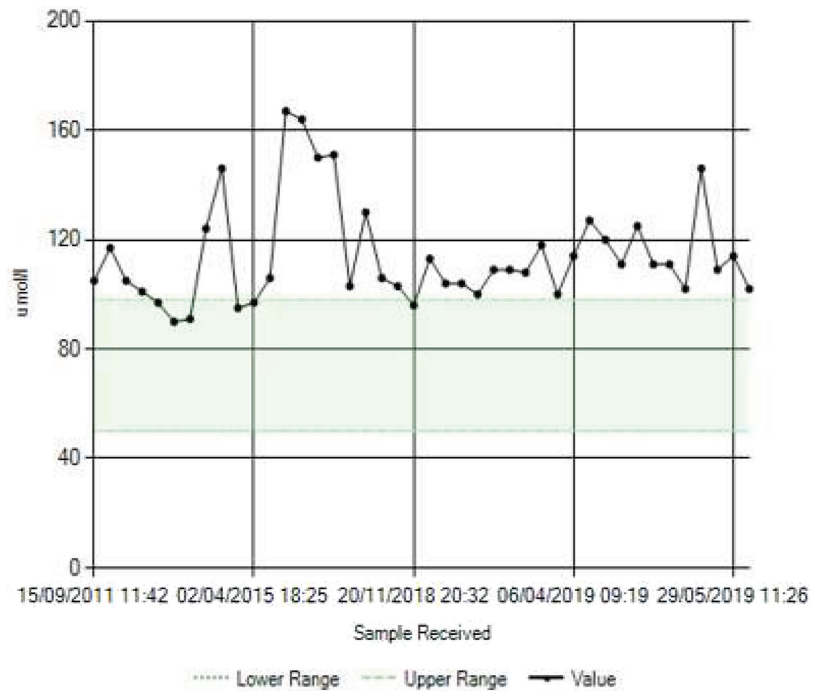

Fig 1. Serum creatinine.

malignancy. Digital subtraction angiography and brain biopsy were both normal. She received steroids empirically on the advice of haematologists. Her platelet counts and renal function improved and atherosclerosis was felt to be the likely cause for her stroke, which was treated conventionally.

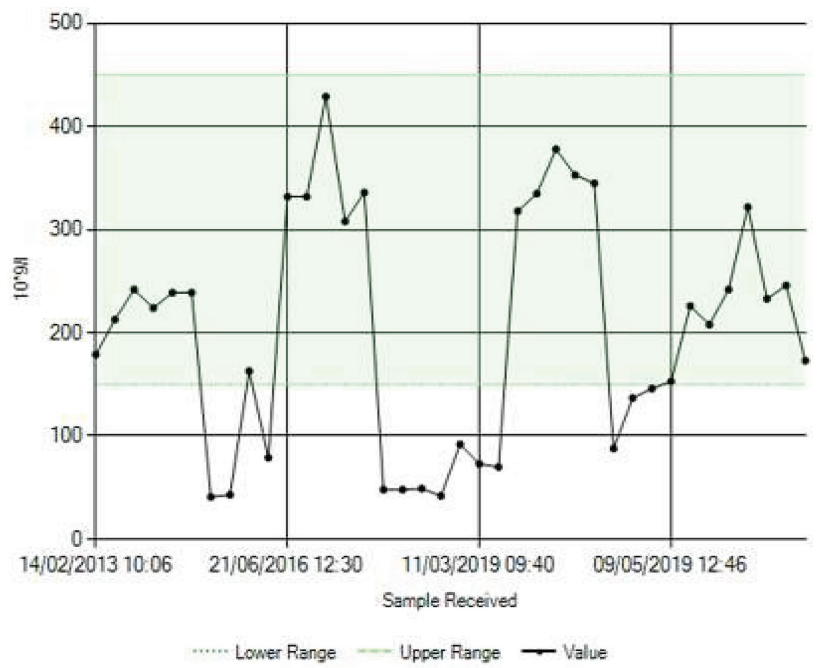

Fig 2. Platelet count. 
She re-presented in 2016 with left arm weakness and speech disturbance. Further strokes in multiple territories were identified on brain magnetic resonance imaging (MRI), although not all were symptomatic. Thrombocytopenia and a high creatinine were noted again. Following a review by the nephrology team, she was deemed to have renal vascular disease. She was commenced on dual antiplatelet agents and blood pressure control was tightened.

Following her latest presentation, there was suspicion of this being an unusual case of thrombotic thrombocytopenic purpura (TTP), which is typically associated with high mortality if left untreated. This was confirmed by undetectable ADAMTS13 assay and high inhibitor levels. She had three out of the five features of the TTP pentad and was induced into remission with plasma exchange and immune suppression. Hence the mystery of the missing 'Edward Scissorhands' (ie ADAMTS13), which cleaves von Willebrand factor multimers, thereby preventing haemolysis and formation of micro thrombi, was solved.

\section{Conclusion}

This unusual case highlights the diagnostic challenges in a patient with cardiovascular risk factors having recurrent strokes.

\section{Conflicts of interest}

None declared. 